Sir,

\section{Corneal endotheliitis with cytomegalovirus infection of corneal stroma}

Although involvement of cytomegalovirus (CMV) in corneal endotheliitis was recently reported, the pathogenesis of this disease remains uncertain. ${ }^{1-8}$ Here, we report a case of corneal endotheliitis with CMV infection in the corneal stroma.

\section{Case}

A 44-year-old man was referred for a gradual decrease in vision with a history of recurrent iritis with unknown aetiology. The corrected visual acuity in his right eye was $20 / 200$. Slit lamp biomicroscopy revealed diffuse corneal oedema with pigmented keratic precipitates (KPs) without anterior chamber cellular reaction (Figure 1a). The patient had undergone penetrating keratoplasty in August 2006, and pathological examination showed nonspecific corneal oedema. At the seventh postoperative week, coin-shaped KPs appeared (Figure 1b).

Administration of oral valacyclovir and frequent topical corticosteroid ( $0.1 \%$ betamethasone) was not effective, and the corneal oedema worsened with an intraocular pressure rise (Figure 1c). Intravenous methylprednisolone $1000 \mathrm{mg}$ per day for 3 days followed by oral betamethasone gave only a temporal improvement. As his blood sugar level increased, we administered oral cyclosporine A with blood level at $2 \mathrm{~h}$ maintained at approximately $800 \mathrm{ng} / \mathrm{ml}$. The treatment resulted in gradual decreases of KPs, but graft oedema persisted. Vision decreased to 20/2000.

The patient underwent a second keratoplasty combined with cataract surgery in August 2007. The aqueous humour was tested for polymerase chain reaction to detect $\mathrm{HSV}, \mathrm{VZV}$, or CMV; a positive result being obtained only for CMV-DNA. Pathological examination demonstrated granular deposits in the deep stroma, which was positive for CMV by immunohistochemistry (Figures $2 \mathrm{a}$ and $\mathrm{b}$ ). The cells showed a typical 'owl's eye' morphology (Figure 2c).

We commenced systemic gancyclovir at $10 \mathrm{mg}$ per day for 7 days, followed by topical $0.5 \%$ gancyclovir eye drops six times a day. With the postoperative follow-up period of 20 months, the graft remained clear without KPs (Figure 1d). The patient has been treated with gancyclovir eye drops t.i.d. to date. His visual acuity improved to 20/20, and endothelial density was $2300 / \mathrm{mm}^{2}$. Repeated PCR in aqueous humour for $\mathrm{CMV}$ yielded a negative result in the 10th week.

\section{Comment}

Involvement of CMV in anterior segment disease was considered rare until recent reports indicated the presence of CMV-DNA in the aqueous humour of patients with corneal endotheliitis. ${ }^{1-8}$ In this case, we detected CMV antigen in the corneal stroma of a patient with corneal endotheliitis. Pathological findings revealed the typical 'owl's eye' appearance in the
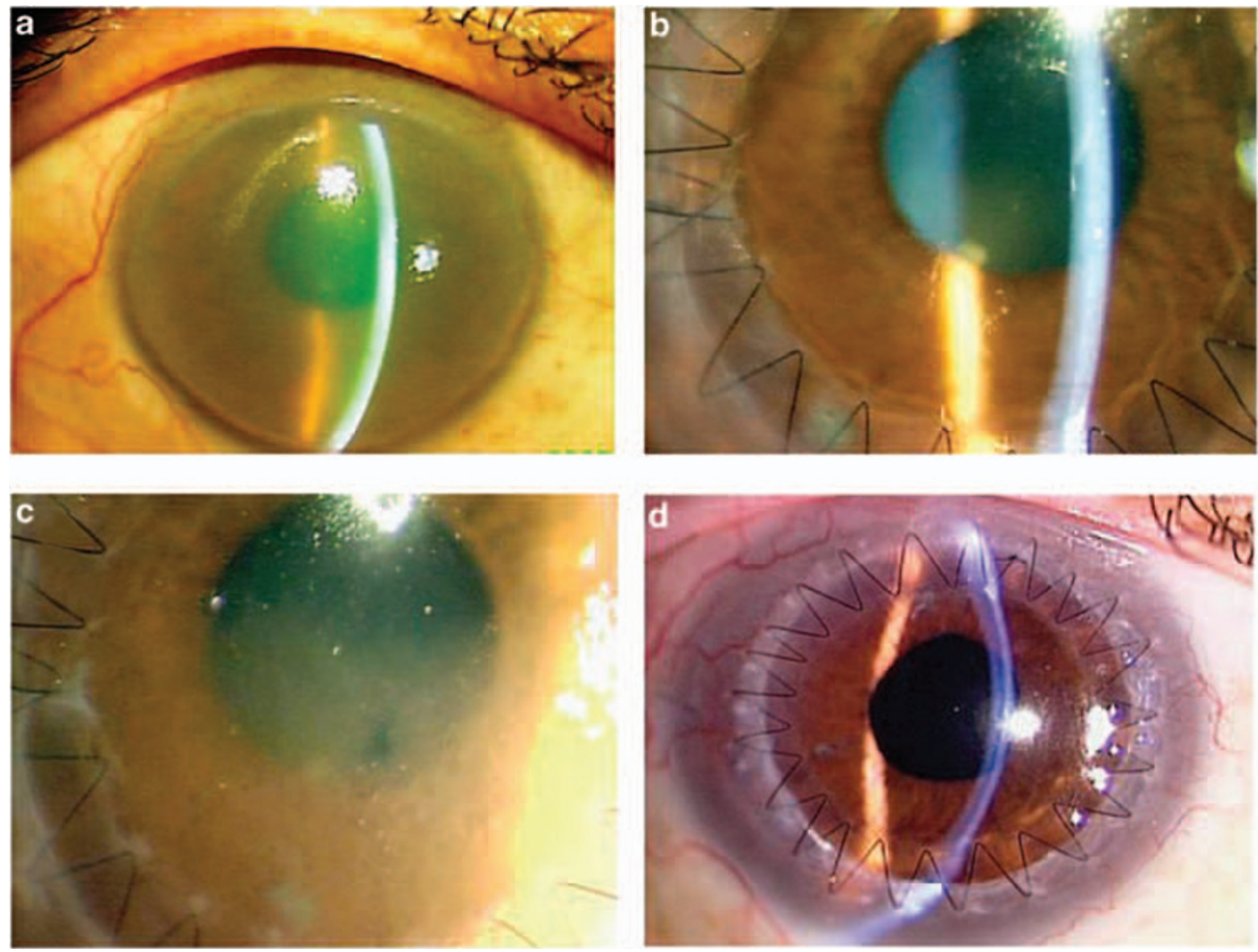

Figure 1 (a) Preoperative cornea. Note diffuse oedema with no active anterior chamber reaction. (b) Coin-shaped keratic precipitates developed 7 weeks after keratoplasty. (c) Corneal stromal oedema with keratic precipitates in the corresponding area spread to the inferior half of the graft. (d) Three months after the second keratoplasty. The graft remained perfectly clear, with no keratic precipitates. 

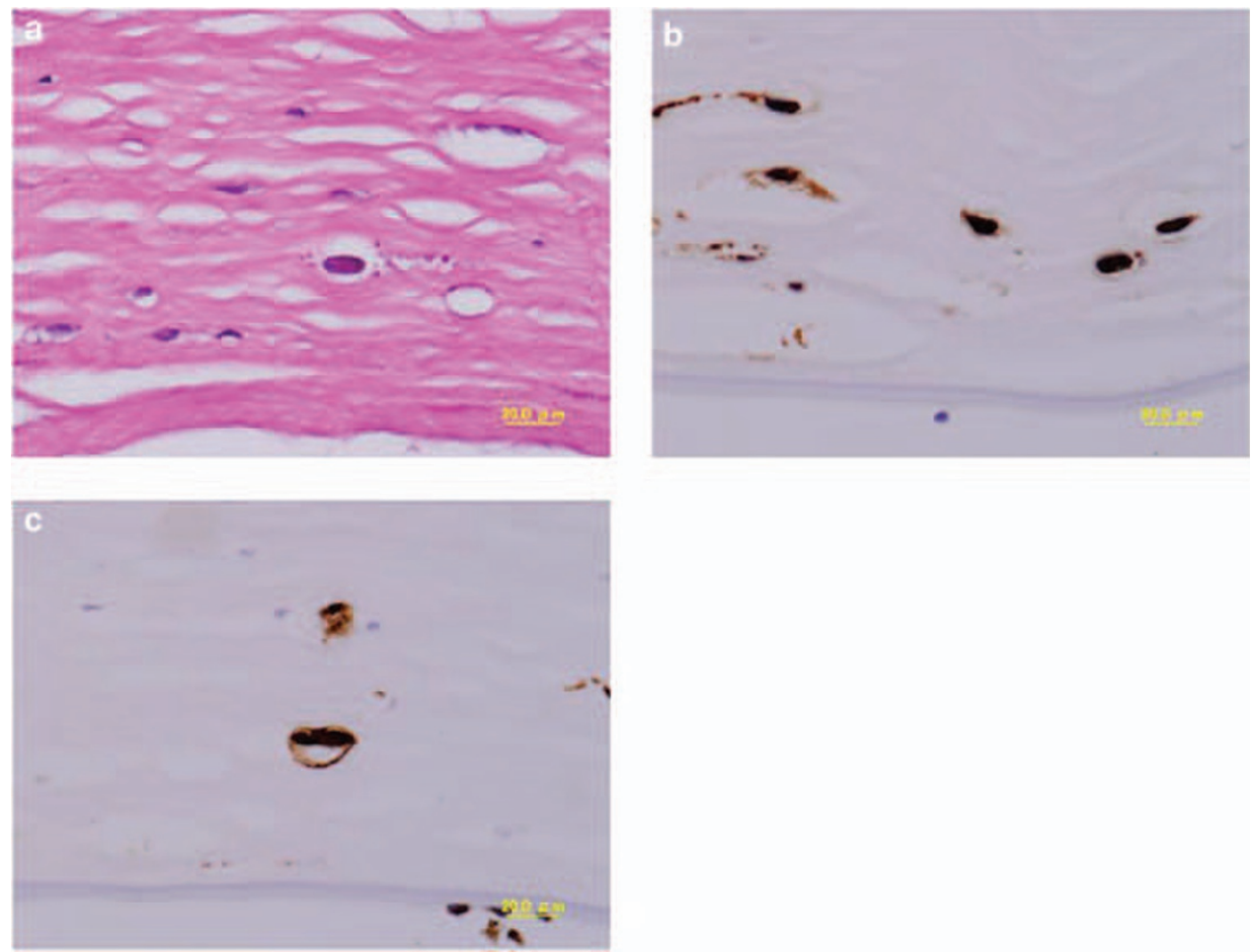

Figure 2 (a) Hematoxylin and eosin staining of the sample demonstrated granular deposits in the deep stroma. (b) Immunohistochemistry for CMV showed positive staining in stromal deposits. (c) Typical 'owl's eye' in the sample.

deep corneal stroma, similar to a previous report using confocal microscopy. ${ }^{9}$ Endotheliitis did not recur with gancyclovir treatment in our case. These findings strongly suggest that CMV infection is the cause of corneal endotheliitis. We did not find histopathological evidence that the corneal endothelium was infected or inflamed by CMV, therefore the present case can be considered as CMV stromal keratouveitis. However, we consider the term 'endotheliitis' to be appropriate to be used in this case because the clinical findings are indistinguishable to the CMV endotheliitis that has been reported. ${ }^{3,4,7,10}$ It is likely that the corneal endothelium was destroyed during the disease process. It is possible that proliferation of CMV resulted from the relatively intense immunosuppression given, as we suspected that endothelial rejection might be caused.

There could be two explanations regarding the origin of CMV in the patient. One is that the patient had CMV infection before the first keratoplasty, and the other is that CMV was acquired after the first grafting, including the possibility of donor-to-recipient infection. It cannot be confirmed at this moment because neither molecular studies on the patient's cornea nor donor screening for CMV has been performed. It seems reasonable to consider that CMV was the cause of recurrent iritis because of the deleterious effect on the corneal endothelium and similarities with previous reports on CMV endotheliitis. ${ }^{3,4,7,10}$

\section{Conflict of interest}

The authors declare no conflict of interest.

\section{Acknowledgements}

We thank Associate Professor Jeremy Williams, Tokyo Dental College, for his help with the English of the paper.

\section{References}

1 Anshu A, Chee SP, Mehta JS, Tan DT. Cytomegalovirus endotheliitis in Descemet's stripping endothelial keratoplasty. Ophthalmology 2009; 116: 624-630.

2 Chee SP, Bacsal K, Jap A, Se-Thoe SY, Cheng CL, Tan BH. Corneal endotheliitis associated with evidence of cytomegalovirus infection. Ophthalmology 2007; 114: 798-803.

3 Hwang YS, Hsiao CH, Tan HY, Chen KJ, Chen TL, Lai CC. Corneal endotheliitis. Ophthalmology 2009; 116: 164-e1.

4 Koizumi N, Suzuki T, Uno T, Chihara H, Shiraishi A, Hara Y et al. Cytomegalovirus as an etiologic factor in corneal endotheliitis. Ophthalmology 2008; 115: 292-297.

5 Koizumi N, Yamasaki K, Kawasaki S, Sotozono C, Inatomi T, Mochida $\mathrm{C}$ et al. Cytomegalovirus in aqueous humor from an eye with corneal endotheliitis. Am J Ophthalmol 2006; 141: 564-565.

6 Ohashi Y, Kinoshita S, Mano T, Kiritoshi A, Ohji M. Idiopathic corneal endotheliopathy. A report of two cases. Arch Ophthalmol 1985; 103: 1666-1668.

7 Suzuki T, Ohashi Y. Corneal endotheliitis. Semin Ophthalmol 2008; 23: 235-240.

8 Yamauchi Y, Suzuki J, Sakai J, Sakamoto S, Iwasaki T, Usui M. A case of hypertensive keratouveitis with endotheliitis associated with cytomegalovirus. Ocul Immunol Inflamm 2007; 15: 399-401.

9 Shiraishi A, Hara Y, Takahashi M, Oka N, Yamaguchi M, Suzuki T et al. Demonstration of 'owl's eye' morphology by 
confocal microscopy in a patient with presumed cytomegalovirus corneal endotheliitis. Am J Ophthalmol 2007; 143: 715-717.

10 Chee SP, Bacsal K, Jap A, Se-Thoe SY, Cheng CL, Tan BH. Clinical features of cytomegalovirus anterior uveitis in immunocompetent patients. Am J Ophthalmol 2008; 145: 834-840.

J Shimazaki ${ }^{1}$, A Harashima ${ }^{1}$ and Y Tanaka

${ }^{1}$ Department of Ophthalmology, Tokyo Dental

College, Chiba, Japan

${ }^{2}$ Division of Surgical Pathology, Tokyo Dental

College, Chiba, Japan

E-mail: jun@eyebank.or.jp

Eye (2010) 24, 1105-1107; doi:10.1038/eye.2009.240; published online 9 October 2009

Sir

The surgical outcome and personality change in a child with congenital cataract after multifocal intraocular lens implantation

A 7-year-old girl first presented in January 2007 with bilateral cortical cataract combined with high hyperopia and astigmatism. The refractions were +3.75 spherical diopter (DS) / -1.75 diopter of cylinder (DC) $\times 175$ in the right eye (OD), and $+4.25 \mathrm{DS} /-2.25 \mathrm{DC} \times 170$ in the left eye (OS). The best-corrected visual acuity (BCVA) was 20/32 in either eye (OU). Spectacles correction and CAM vision-stimulator treatment once a week were prescribed, and BCVA in OU reached 20/25 after 5 months.

However, the cortical opacity (OU) aggravated gradually. As the BCVA decreased to 20/40 OD and 20/70 OS in January 2009, bilateral simultaneous phacoemulsification with multifocal IOLs-Tecnis MF (ZM900, AMO) implantation OU was performed in February. The refractions were $+1.25 \mathrm{DS} /-2.25 \mathrm{DC} \times 170 \mathrm{OD}$ and $+1.0 \mathrm{DS} /-2.75 \mathrm{DC} \times 175$ OS 4 weeks after surgery, and the BCVA was 20/30 OU. New spectacles were prescribed and 6 months later the BCVA reached 20/20 OU without performing the CAM treatment after surgery.

According to the statement of the parents, the girl had become introverted and shrinking while vision decreased. However, the girl is vigorous now and is willing to participate in outdoor activities. The Children's Visual Function Questionnaire (CVFQ) developed by Felius et al is an instrument designed for parents of children aged $\leqslant 7$ years to assess the influence of children's visual disorders on themselves and on their families. ${ }^{1}$ The CVFQ was filled by the girl's parents before and 6 months after surgery, and the CVFQ total score and subscale scores represented better results (higher scores) after surgery, except for the general health subscale (Table 1). We thought that in the assessment of cataract in children, not only the visual acuity but also the quality of children's life is the concern at the time of surgical intervention. In recent years, new surgery techniques, designed IOLs, and IOL materials have proved to yield good results in senile cataract surgery.
Table 1 The subscale scores of the Children's Visual Function Questionnaire

\begin{tabular}{lcc}
\hline & Pre-operation & Post-operation \\
\hline General health & 0.75 & 0.75 \\
General vision & 0.2 & 0.4 \\
Competence & 0.6 & 0.95 \\
Personality & 0.89 & 0.97 \\
Family impact & 0.25 & 0.54 \\
Treatment & 0.25 & 0.75 \\
Total score & 0.54 & 0.82 \\
\hline
\end{tabular}

These advances have also increased the success rate of cataract surgery in children., ${ }^{2,3}$ In this case, the cataract extraction and multifocal IOL implantation were successful in treating the amblyopia and in reshaping the patient's personality.

\section{Conflict of interest}

The authors declare no conflict of interest.

\section{References}

1 Felius J, Stager Sr D, Berry P, Fawcett SL, Stager Jr DR, Salomão SR et al. Development of an instrument to assess vision-related quality of life in young children. Am J Ophthalmol 2004; 138: 362-372.

2 Jacobi PC, Dietlein TS, Konen W. Multifocal intraocular lens implantation in pediatric cataract surgery. Ophthalmology 2001; 108: 1375-1380.

3 Wilson Jr ME, Bartholomew LR, Trivedi RH. Pediatric cataract surgery and intraocular lens implantation: practice styles and preferences of the 2001 ASCRS and AAPOS memberships. J Cataract Refract Surg 2003; 29: 1811-1820.

$\mathrm{H}-\mathrm{Y} \operatorname{Lin}^{1,2}, \mathrm{C}-\mathrm{E}$ Wang ${ }^{1}, \mathrm{~S}-\mathrm{Y} \mathrm{Lin}^{3}$ and $\mathrm{H}-\mathrm{K} \mathrm{Hsu}^{4}$

${ }^{1}$ Universal Eye Center, Zhongli Branch, Taiwan

${ }^{2}$ Department of Optometry, Central Taiwan

University of Science and Technology, Taichung, Taiwan

${ }^{3}$ Department of Ophthalmology, Cathay General Hospital, Taipei, Taiwan

${ }^{4}$ Universal Eye Center, Xinzhuang Branch, Taiwan

E-mail: hsu-eye@hotmail.com

Eye (2010) 24, 1107; doi:10.1038/eye.2009.293; published online 11 December 2009

Sir,

Myopic pre-foveoschisis: an earlier stage of myopic foveoschisis documented by optical coherence tomography

Myopic foveoschisis (MF) occurs in $9-34 \%$ of highly myopic staphylomatous eyes, ${ }^{1-3}$ which may be associated with or may progress to foveal detachment or macular hole, which in turn are associated with decreased visual 